\title{
Compact low loss MEMS phase shifters for scalable field-programmable silicon photonics
}

\author{
Pierre Edinger ${ }^{1}$, Carlos Errando-Herranz ${ }^{1}$, Alain Yuji Takabayashi ${ }^{2}$, Hamed Sattari $^{2}$, \\ Niels Quack $^{2}$, Peter Verheyen ${ }^{3}$, Wim Bogaerts ${ }^{3,4}$, Kristinn B. Gylfason ${ }^{1}$ \\ ${ }^{1}$ KTH Royal Institute of Technology, Stockholm, Sweden; ${ }^{2}$ Ecole Polytechnique Federale de Lausanne, \\ Switzerland; ${ }^{3}$ Interuniversity Microelectronics Centre, Leuven, Belgium; ${ }^{4}$ Ghent University, Belgium \\ edinger@kth.se
}

\begin{abstract}
MEMS offer low power tunability to silicon photonics. However, reported phase shifters lack in range, IL, or linearity. We show $\pi$ linear phase shift in compact, 0.2 dB-IL MEMS devices, and demonstrate trade-offs for scalability. (C) 2020 The Author(s)
\end{abstract}

\section{Introduction}

Application specific silicon photonic integrated circuits (PICs) are very successful, e.g. in transceivers for datacenters, and rapid progress is being made on field-programmable silicon photonics [1]. However, the mW power consumption of the thermal active components commonly employed prohibits scaling to large circuits. Microelectromechanical Systems (MEMS) offer $10^{4}$ lower power consumption, and silicon has excellent mechanical properties. This combination is particularly attractive, since the same silicon device layer can be used for both the photonic waveguides and the electro-mechanical actuators. Out-of-plane and in-plane displacement of silicon waveguides, rims, or membranes has been reported for phase shifting [2-4]. However, the devices shown so far have limited scalability, either due to high losses [2,3] or limited phase shifts [4].

\section{Design and fabrication}

Here, we use in-plane displacement of a slender suspended silicon rim to tune the effective mode index of a fixed waveguide (Fig. 1 (a-b)). The rim is pulled away from the waveguide using a $40 \times 30 \mu \mathrm{m}^{2}$ comb-drive actuator, thereby reducing its overlap with the evanescent field of the waveguide mode. Waveguide bends and folded springs reduce buckling due to residual device layer stress, Fig. 1 (c). Three phase shifters with waveguide widths of 350 , 400 and $450 \mathrm{~nm}$, for devices I-III, respectively, were made in order to evaluate the trade-off between tuning range and insertion loss. All devices use the same electrostatic comb-drive actuator.
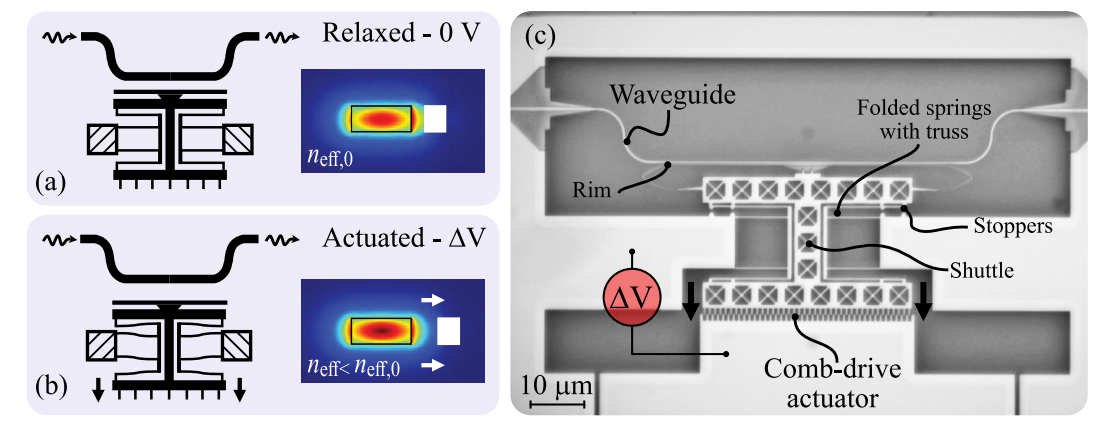

Fig. 1. Our phase shifter design. In (a-b), illustration of the $n_{\text {eff }}$ tuning principle. In (c), microscope top view of a fabricated device. The three devices presented in this work only differ in waveguide width, and look identical under the microscope.

The devices were fabricated in IMEC's iSiPP50G platform, but the tests reported here were done on early samples without any metallization. The final step of MEMS device release was performed by post-processing at the Center of MicroNanoTechnology (CMi) at EPFL. 


\section{Measurements}

The phase shifters were included in one arm of integrated unbalanced Mach-Zehnder interferometers, and the wavelength shift of the transmission spectrum (Fig. 2 (a-c)) was used to extract phase shift as a function of voltage (Fig. 2 (d)). Additionally, the insertion loss (IL) was extracted for all three devices (Fig. 2 (e)). The IL values were extracted from the measured extinction ratios and by comparison with a straight suspended reference waveguide. Between each voltage step, an additional measurement at $0 \mathrm{~V}$ was done to confirm hysteresis-free actuation.
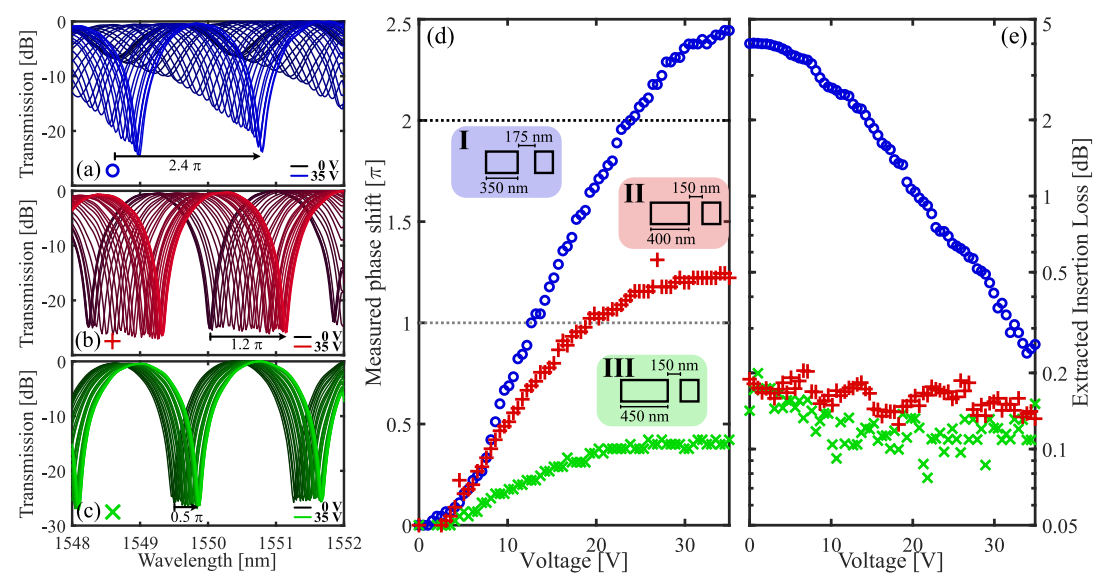

Fig. 2. Measurement results. In (a-c), measured spectra against voltage for three different waveguide geometries. (a) Device I: width $350 \mathrm{~nm}$, gap $175 \mathrm{~nm}$. (b) Device II: width $400 \mathrm{~nm}$, gap $150 \mathrm{~nm}$. (c) Device III: width $450 \mathrm{~nm}$, gap $150 \mathrm{~nm}$. (d) Extracted phase shift, and (e) IL at $\lambda=1550 \mathrm{~nm}$.

As intended, the devices achieve good linearity, since the $V^{2}$ dependence of the displacement of the comb-drive actuator balances out the exponential dependence of the effective index on the gap. Phase shifter I attains $2.4 \pi$ phase shift, but at the expense of high variable IL. On the other hand, phase shifter III reaches a lower phase shift of $0.4 \pi$, but has a very low and stable IL. All devices show improved performance compared to previous results [2-5], but the trade-offs can be used to target specific scaling, which can be footprint or IL limited [5]. Device II, for instance, offers very good balance in scaling figures: linear, $\pi$ phase shift with $20 \mathrm{~V}$ and $0.2 \mathrm{~dB}$ IL.

\section{Conclusions}

We have designed and measured MEMS phase shifters with high linearity, tunability and low IL on chips from a silicon photonics foundry, and analyzed their scalability trade-offs. We believe that both the devices' performance and derived trade-off analysis are a significant step towards foundry-ready large-scale silicon photonic MEMS. In particular, we demonstrate linear $\pi$ tuning in a $40 \times 30 \mu \mathrm{m}^{2}$ footprint phase shifter with $0.2 \mathrm{~dB}$ IL.

\section{Acknowledgements}

This work has received funding from the European Union's Horizon 2020 research and innovation programme under grant agreement No.780283 (MORPHIC). We thank Dr. Max Yan for access to measurement equipment.

\section{References}

1. D. Pérez, I. Gasulla, L. Crudgington, D. J. Thomson, A. Z. Khokhar, K. Li, W. Cao, G. Z. Mashanovich, and J. Capmany, "Multipurpose silicon photonics signal processor core," Nature Communications, vol. 8, p. 636, Sept. 2017.

2. T. Ikeda, K. Takahashi, Y. Kanamori, and K. Hane, "Phase-shifter using submicron silicon waveguide couplers with ultra-small electro-mechanical actuator," Optics Express, vol. 18, pp. 7031-7037, Mar. 2010.

3. K. V. Acoleyen, J. Roels, P. Mechet, T. Claes, D. V. Thourhout, and R. Baets, "Ultracompact Phase Modulator Based on a Cascade of NEMS-Operated Slot Waveguides Fabricated in Silicon-on-Insulator," IEEE Photonics Journal, vol. 4, pp. 779-788, June 2012.

4. C. Errando-Herranz, F. Niklaus, G. Stemme, and K. B. Gylfason, "Low-power microelectromechanically tunable silicon photonic ring resonator add-drop filter," Optics letters, vol. 40, no. 15, pp. 3556-3559, 2015.

5. C. Errando-Herranz, A. Y. Takabayashi, P. Edinger, H. Sattari, K. B. Gylfason, and N. Quack, "MEMS for Photonic Integrated Circuits," IEEE Journal of Selected Topics in Quantum Electronics, vol. 26, pp. 1-16, Mar. 2020. 